\title{
El acto de habla oferta en la publicidad digital de viajes en francés ${ }^{1}$
}

\author{
The speech act of offering in digital \\ travel advertising in French
}

\author{
Arrate Aldama Epelde \\ arratealdama@gmail.com \\ Universidad del País Vasco
}

\begin{abstract}
The pragmatic study of speech acts has undergone a considerable evolution in recent years. In the light of the influence of new technologies on society, it is necessary to study speech acts in the field of digital discourse, in order to account the current forms of communication. Bearing this reality in mind, this article aims to analyse offering as a speech act in a specific context and mass media: digital travel advertising in French. We analyse specifically the linguistic strategies of the offering in a corpus of travel offers, as well as their characteristics and the external modification that accompanies them, with the purpose of showing a renewed study on speech acts, in general, and on the offering, in particular, and performing new considerations on this speech act in the context of the electronic advertising.
\end{abstract}

\section{Key-words}

offering, speech act, advertising, travels, Internet.

\begin{abstract}
Résumé
L'étude pragmatique sur les actes de parole a connu une évolution considérable ces dernières années. Vu que les nouvelles technologies ont de l'influence sur la société, il est nécessaire d'étudier les actes de parole dans le domaine du discours numérique pour rendre compte des formes actuelles de communication. En considérant cette réalité, l'objectif de cet article est d'analyser l'acte d'offrir dans un contexte et un média spécifiques : la publicité numérique de voyage en français. Concrètement, nous étudierons les stratégies linguistiques de l'acte d'offrir d'un corpus d'offres de voyage, ainsi que leurs caractéristiques et la modification externe qui les accompagne, afin de présenter une étude novatrice sur les actes de parole, en général, et sur l'offre, en particulier, et formuler de nouvelles considérations sur cet acte de parole dans le cadre de la publicité sur Internet.
\end{abstract}

\section{Mots-clés}

offre, acte de parole, publicité, voyages, Internet.

1 Este artículo se enmarca en el proyecto de investigación FFI2015-63715, financiado por el Ministerio de Economía y Competitividad (MINECO) y el Fondo Europeo de Desarrollo Regional (FEDER). 


\section{Introducción}

El estudio de los actos de habla ha sido y sigue siendo una línea de investigación de gran relevancia en pragmática. Desde los trabajos de Austin $(1975)$ y Searle $(1969,1979)$ hasta la actualidad, la teoría de los actos de habla ha conocido una importante evolución que atañe no solo a la propia teoría en sí, sino también a los trabajos realizados en distintas lenguas. La mayoría de estos trabajos se han llevado a cabo en inglés, ya que la pragmática nació en ámbito anglófono. Aunque estos han servido primero de modelo a estudios en lenguas europeas y, posteriormente, a lenguas asiáticas, como el chino o el japonés.

A día de hoy, el estudio de los actos de habla se ha extendido a nuevos contextos comunicativos, de acuerdo con las nuevas formas que tienen los hablantes para comunicarse entre sí. Los lingüistas han pasado de analizar los actos de habla en interacciones verbales cara a cara entre hablantes a estudiarlos en el discurso de Internet. Un medio, el de Internet, que rige por completo las relaciones humanas y combina rasgos del discurso oral y escrito para dar lugar a lo que se conoce como discurso digital.

Esta nueva realidad en el análisis de los actos verbales nos lleva a escoger como contexto el medio electrónico, ya que los estudios de actos de habla en este nuevo contexto empiezan a proliferar. Nos hemos decantado además por la oferta porque es un acto de habla poco estudiado en general, y poco estudiado en francés, a pesar de que está presente en nuestra vida cotidiana y en los medios de comunicación. Y hemos decidido analizar este acto verbal en la publicidad de viajes en francés de las páginas web de agencias de viajes por tratarse del contexto en el que más se explota. Entendemos que este medio de comunicación y este contexto específicos nos permitirán abordar un análisis renovado del acto de habla oferta.

Para ello, definimos en primer lugar las características de este acto basándonos en los trabajos enmarcados en la teoría de los actos de habla y los estudios específicos sobre la oferta. A continuación, presentamos el corpus que hemos confeccionado para llevar a cabo este estudio. Partiendo de las características del acto de ofrecer y del corpus creado, confeccionamos una clasificación de las estrategias lingüísticas empleadas en el discurso verbal de nuestras ofertas de viajes. Finalmente, llevamos a cabo el estudio del acto de habla oferta. Hemos decidido analizar primero el acto de habla director y luego la modificación, las dos partes de las que se compone la secuencia de oferta. Dentro del acto de habla director, estudiamos las estrategias lingüísticas utilizadas en la consecución del acto, así como los rasgos fundamentales de dichas estrategias. Y, dentro de la modificación, analizamos la modificación externa, es decir, los procedimientos que preceden o suceden al acto de habla director para completarlo o variar su significado (Pohle, 2009). 
Anales de Filología Francesa, n. ${ }^{\circ}$ 28, 2020

Arrate Aldama Epelde

\section{El acto de habla oferta en pragmática}

El acto de habla oferta ha sido analizado en pragmática tanto por trabajos enmarcados en la línea de la teoría de los actos de habla como por trabajos específicos sobre la oferta.

Dentro de la teoría de los actos de habla, Austin (1975) afirma en principio que ofrecer es un acto ejercitativo; es decir, un acto que sirve para emitir un juicio a favor o en contra de una forma de actuar, o para inducir a los demás a comportarse de una manera o de otra. Añade que los ejercitativos como ofrecer están sujetos a una conducta concreta: cuando un hablante realiza una oferta, la intención de ese acto es la de comprometer personalmente a ese hablante con una conducta (la de ofrecer algo a alguien). Por lo que, posteriormente, considera que ofrecer también puede pertenecer a la clase de los comisivos. Estos actos se definen como aquellos que obligan al hablante a adoptar una cierta conducta: cuando el hablante ofrece algo al oyente, está obligado a cumplir con su palabra.

En trabajos posteriores, se produce un gran debate por determinar la naturaleza de la oferta. Autores como Searle $(1969,1979)$ o Bach y Harnish (1979) mantienen que es un acto comisivo, esto es, una acción futura que parte de la iniciativa del locutor y que este se compromete a llevar a cabo, lo que hace que adquiera una obligación. Pero dicho acto se cumple de manera satisfactoria, siempre y cuando lo ofertado resulte del interés del interlocutor, beneficiario de la oferta. Hancher (1979), por su parte, defiende que es un acto comisivo y directivo, y crea además la clase de actos ilocucionarios "comisivos-directivos". Según su teoría, ofrecer algo a alguien es intentar influir en el comportamiento de los demás (acto directivo) y realizar la acción que cambie dicho comportamiento (acto comisivo).

A pesar de las diferencias, estos trabajos coinciden en que existen tres aspectos que definen la oferta. Cuando el hablante realiza una oferta al oyente:
a. El hablante expresa su buena voluntad para llevar a cabo la acción.
b. El hablante asume que al emitir el enunciado está en la obligación de llevar a cabo la acción.
c. La acción interesa al oyente.

Los estudios de pragmática sobre la oferta también sostienen que este acto verbal es de carácter comisivo y directivo. Aijmer (1996) aboga por el carácter comisivo y directivo de la oferta en la misma proporción, y Pérez Hernández (2001a, 2001b), en base a su Modelo Proposicional Cognitivo Idealizado, la define también como un acto ambivalente. Pero dentro de ese continuum comisivo-directivo, la sitúa muy próxima a actos prototípicamente comisivos. Barron (2005) contempla, en su análisis comparativo en dos variedades lingüísticas del inglés, que la oferta emplea estrategias comisivas y directivas, mostrando la naturaleza confusa de este acto de habla (fuzzy nature). Y, Pohle (2009), en su elaborada tesis doctoral sobre la oferta en las negociaciones empresariales en el inglés de Irlanda, manifiesta que las 
ofertas resultan comisivas y, al mismo tiempo, condicionales, ya que la responsabilidad del locutor en el acto está condicionada por la aceptación del interlocutor, y directivas, puesto que buscan una reacción por parte del interlocutor.

Observamos que se produce una evolución en el análisis sobre la naturaleza del acto de habla oferta: si bien en un principio solo se acepta que la oferta es un acto comisivo, después se tiene en cuenta su carácter comisivo y directivo.

Las consideraciones realizadas sobre el acto de ofrecer por todos estos trabajos resultan pues muy necesarias para poder llevar a cabo nuestro estudio. Para ello, creamos previamente el corpus y la clasificación de las ofertas de viajes, que presentamos seguidamente.

\section{Corpus de las ofertas de viajes}

Nuestro corpus está compuesto por 550 ofertas redactadas en francés que han sido tomadas de las páginas web de 43 agencias de viajes ${ }^{1}$ que operan en Internet, para que el estudio quede fundamentado.

En la tarea de recogida de muestras, hemos buscado que todas las agencias de viajes tengan la misma representación en nuestro corpus, por lo que hemos tomado entre 12 y 14 ofertas de cada una de ellas. Y hemos tenido en cuenta además que las ofertas presentaran un texto de una extensión de entre uno y seis enunciados máximo, para que la oferta no resultara demasiado extensa.

Si bien hemos considerado dos factores como la equidad en el número de ofertas seleccionadas por página web y la extensión del texto lingüístico de estas, su elección ha sido aleatoria. Hemos buscado variedad en cuanto al destino vacacional (países, ciudades o islas) y al tipo de viaje (circuitos, cruceros, estancias cortas o de fin de semana...). Hemos configurado así un corpus auténtico, muy diverso y representativo.

Las muestras han sido recogidas respetando su diseño y distribución. Todas o la mayoría de ellas contienen los mismos elementos: un título, una imagen del destino vacacional y un texto escrito. Tanto el título como el texto se valen de la tipografía (tamaño y tipo de letra, negrita o cursiva) como táctica para llamar la atención del lector y atraerlo a la oferta. Se entiende pues que el discurso electrónico es de naturaleza textual e icónica (Bolaños et al., 2004). Sin embargo, debemos puntualizar que en nuestro estudio nos centramos en el texto escrito para estudiar el acto de habla de las ofertas publicitarias de viajes de Internet.

Además de estas consideraciones sobre el contenido de las ofertas de nuestro corpus, debemos tener muy presente el contexto (la publicidad de viajes) y el medio (Internet) en los que se producen para abordar su estudio, ya que les confieren una característica muy particular: en nuestras ofertas de viajes, no se produce una respuesta verbal del interlocutor a la oferta propuesta por el locutor. El locutor y el interlocutor no se encuentran en el mismo lugar

1 Hemos añadido un listado de los enlaces a las páginas web de las agencias de viajes en un anexo al final del artículo. 
y al mismo tiempo cuando se produce la oferta como sucede en las interacciones verbales cara a cara. Esta situación imposibilita una interacción verbal en tiempo real. Además, por el contexto comunicativo en el que se llevan a cabo las ofertas, no se espera una respuesta verbal por parte del interlocutor. La respuesta, en todo caso, sería contratar el viaje. Este tipo de respuesta no nos permite conocer las reacciones de los destinatarios ante lo que han leído e interpretar si los destinatarios han comprendido que se trata de una oferta (Conein, 1985: 113, 117). Deberíamos asumir que la oferta no existe si no hay respuesta del destinatario, ya que Conein (1986) asegura que el recurso a la réplica es constitutivo del acto de ofrecer e invitar. Aun así, mantenemos que en este tipo de publicidad sí se lleva a cabo el acto de ofrecer. Pese a que no hay una reacción verbal, el locutor sí obtiene una respuesta no verbal en forma de aceptación, de rechazo o de indiferencia o no respuesta.

De acuerdo con el método Blum-Kulka et al. (1989), aún considerado un referente en materia de análisis de actos de habla, hemos extraído el acto de habla director del texto de las ofertas, y lo hemos separado de sus expansiones anteriores y posteriores (modificación externa). De esta forma estudiamos tanto el acto de habla director como la modificación externa del texto de nuestras ofertas de viajes. Pero antes de llevar a cabo dicho estudio realizamos una clasificación de las estrategias lingüísticas utilizadas en el acto de habla director.

\section{Clasificación de las estrategias lingüísticas empleadas en el acto de habla director}

A partir del corpus creado para esta investigación, hemos constituido una clasificación que permite distribuir las principales estrategias lingüísticas utilizadas en la realización de las ofertas de viajes. Hemos considerado los modelos de Schneider (2003) y Barron (2003, 2005) que ordenan y organizan las realizaciones de oferta en dos ámbitos muy diferentes. Schneider (2003) analiza las estrategias típicas de realización de ofertas de hospitalidad y, Barron (2003, 2005), las competencias pragmáticas de un grupo de estudiantes que emplean como actos de habla la petición, la oferta y la reoferta (ofertas de asistencia y de hospitalidad), y el rechazo de las ofertas. En ambos, queda demostrada la naturaleza ambivalente de este acto. Según Barron (2003), la oferta puede ser comisiva en las estrategias de ejecución, como en el ejemplo (1), directiva en las estrategias directivas, como en (2), o condicional en las estrategias de preferencia, como en (3).

(1) I could offer some help.

(2) Come in and have a cuppa.

(3) Would you like me to help you?

En nuestro caso, si bien hemos partido de la orientación de estos dos autores, el análisis de nuestro corpus nos ha llevado a configurar una clasificación propia y muy diferente a la formulada por ellos. Observamos que las agencias de viajes, como locutores, emplean una 
serie de estrategias lingüísticas que contienen una fuerza ilocucionaria diferente para llevar a cabo el acto de ofrecer en el ámbito de la publicidad de viajes de Internet (Searle, 1969, 1979; Bach y Harnish, 1979; López de Lizaga, 2015). En vez de considerar la naturaleza de los enunciados empleados para clasificar las estrategias, nos hemos decantado por su fuerza ilocucionaria, lo que también nos ha permitido asignar un nombre a cada una. Como se puede ver en el siguiente cuadro, nuestra clasificación consta de siete estrategias y dos de ellas, la oferta explícita y las reofertas, contienen tres subestrategias.

\section{El anuncio de la oferta}

2. La oferta explícita

2.1. Con el verbo offrir

2.2. Con el verbo proposer

2.3. Con el verbo inviter

3. La oferta futura

4. La conminación a la oferta

5. La presentación de la oferta

6. La oferta posibilitadora

7. Reofertas

7.1. Subestrategia A

7.2. Subestrategia B

7.3. Subestrategia C

Cuadro 1. Nuestra clasificación de las estrategias lingüísticas de la oferta

Una vez presentadas esquemáticamente las estrategias empleadas por las agencias de viajes en el acto de habla director, procedemos a estudiarlas a continuación.

\section{Análisis de las estrategias lingüísticas empleadas en el acto de habla director}

Pese al orden en que presentamos las estrategias lingüísticas en nuestra clasificación, su uso nos lleva a estudiar primero aquellas que son más numerosas en nuestro corpus y, después, aquellas menos numerosas. Las más abundantes son la oferta futura $(28.5 \%)$, la conminación a la oferta (25.3\%) y las reofertas (23.1\%). Tras ellas, se sitúa la oferta explícita (10.7\%), una estrategia que por lo general es muy poco habitual entre las expresiones de oferta en francés (Roulet, 1977; Conein, 1985, 1986; Ruiz de Zarobe, 2000). Y, por último, 
tenemos tres que, por su escaso protagonismo en el corpus de ofertas, se presentan como estrategias secundarias: la presentación de la oferta (5.5\%), el anuncio de la oferta $(3.5 \%)$ y la oferta posibilitadora (3.5\%). Comenzamos pues a analizar la oferta futura.

\subsection{La oferta futura}

La oferta futura utiliza enunciados declarativos cuyo rasgo más característico son los verbos principales: verbos conjugados en futuro simple de indicativo activo (4), en presente de indicativo (5) y en futuro simple de indicativo pasivo (6).

(4) Hautes dunes ocre et paysages contrastés... La Namibie vous surprendra!

(5) Implanté dans le pays des châteaux et des vignobles, ce tout nouveau club vous attend pour d'inoubliables randonnées en forêt, d'épiques parties de golf et de douces balades sur la Loire.

(6) Vous serez fascinés par l'Islande, terre de glace et de feu où se déclinent tous les phénomènes volcaniques!

Esta estrategia presenta la oferta como una acción futura del locutor en beneficio del interlocutor. Esta definición coincide con las asunciones realizadas por algunos estudios sobre este acto verbal (Searle, 1979; Bach y Harnish, 1979; Conein, 1985, 1986; Pérez Hernández, 2001a, 2002b; Pohle, 2009). En algunas muestras pertenecientes a este procedimiento $(28.7 \%)$, la oferta también resulta un acto futuro que lleva a cabo el interlocutor, representado por el deíctico vous, para su propio beneficio ((7) y (8)).

(7) Vous découvrirez toutes les splendeurs de la Birmanie et ses incontournables lieux : Yangon, Inle, Mandalay, Bagan... et entre ces 2 villages la vie locale rythmée par l'Irrawady.

(8) L'Écosse en moto, après la traversée rapide de l'Irlande, vous rejoignez l'Écosse et les Highlands, paysages sauvages et arides.

Estos parámetros son los mismos que definen la invitación (Tsui, 1994; Ruiz de Zarobe, 2000-2001). Pero, en este caso, no hablamos de que se invita al cliente, ya que no son formulaciones habituales de invitación en francés (Ruiz de Zarobe, 2000-2001), sino que se intenta implicar al cliente en la oferta, convirtiéndolo en agente de la acción. Estas formulaciones representan pues una de las novedades que confiere nuestro trabajo al estudio del acto de ofrecer.

En el $22.9 \%$ de las muestras, las ofertas emplean varios enunciados que plantean el acto de ofrecer como una acción futura del locutor y del interlocutor en una misma secuencia.

(9) Un itinéraire conçu pour les budgets serrés ou les amoureux du camping et qui vous conduira sur les plus beaux sites de Corse. De superbes routes où se succèdent lignes droites et virages à n'en plus finir dans un cadre époustouflant. Vous serez étonnés de la 
multiplicité des paysages, de la gastronomie, de l'accueil qui vous sera réservé... Basés en Corse, nous sillonnons régulièrement toutes les petites routes et vous garantissons un itinéraire exceptionnel!

(10)Des lacs arborés du nord aux dunes de sable du désert de Gobi en passant par les grandes steppes du centre, vous découvrirez durant cette aventure de trois semaines les plus beaux paysages de Mongolie. Voyage d'exception à taille humaine, ce circuit vous offrira également une occasion unique de partager le quotidien d'une famille d'éleveurs nomades. Logé en bivouac et en yourte, vous serez accueilli avec chaleur et simplicité afin de vivre une réelle immersion, en terre inconnue.

Observamos que esta estrategia da gran importancia tanto a los verbos presentes en las secuencias del acto de ofrecer como a la orientación de las mismas puesto que la oferta se plantea como un acto futuro realizado por el locutor o el interlocutor, representado por el deíctico vous, para su propio beneficio.

\subsection{La conminación a la oferta}

La conminación a la oferta también alude al acto de ofrecer como una acción que llevará a cabo el interlocutor en un futuro cercano. Pero destaca sobre todo por ser una táctica muy efectiva de la agencia de viajes para apelar directamente al cliente e inducirle a aceptar lo que se le ofrece (Mansor y Ridruejo, 2014). Para ello, el locutor se vale de enunciados imperativos.

(11) Profitez de vacances animées dans un cadre authentique au riche patrimoine historique.

(12) Vivez Séville dans un palais andalou.

Su efectividad se debe a que manifiesta de manera más contundente que en el resto de las estrategias tres de las intenciones comunicativas de las agencias de viajes (Bhatia, 1993): resulta muy persuasiva, apela directamente al interlocutor para mostrarle las bondades de la oferta y sirve para que el locutor pueda establecer contacto con el interlocutor. En algunas ofertas (15.1\%), ese deseo de vender lleva al locutor a insistir sobre la oferta inicial, utilizando varios enunciados coordinados (13) o yuxtapuestos (14) en los que se emplean verbos en imperativo.

(13) À $10 \mathrm{~km}$ au sud de Cecina, sur la Côte des Étrusques, Marina Di Bibbona est une station balnéaire moderne aux larges plages bordées de forêts de pins. Découvrez l'arrière-pays, idéal pour des promenades en tous genres (VTT, cheval...) et visitez les célèbres villes de San Gimignano Ou Volterra.

(14) Au volant de votre voiture, sillonnez les routes et les parcs du Far West, découvrez le faste délirant de Las Vegas et le charme de San Francisco. 
Tanta insistencia pone de relieve una de las intenciones comunicativas de la agencia: el deseo de convencer al público para que adquiera el viaje.

\subsection{Las reofertas}

La estrategia que sin duda mejor refleja dicha insistencia del locutor en las secuencias de oferta para cumplir sus objetivos comerciales es la relativa a las reofertas. Se sitúa como la tercera más numerosa de nuestro corpus.

En teoría, la reoferta se formula una vez que la oferta inicial ha sido rechazada para intentar convencer al otro de que acepte (Kerbrat-Orecchioni, 2004). Este supuesto se basa en que la secuencia de oferta se produce en una interacción verbal cara a cara entre dos hablantes. Pero en las ofertas de nuestro corpus no existe dicha interacción entre locutor e interlocutor de manera que no hay reacción a la oferta inicial que conlleve una nueva tentativa de oferta. En nuestras ofertas de viajes, los objetivos comerciales de la agencia le llevan a insistir sobre la oferta inicial y realizar reofertas que están unidas entre sí mediante conectores y anáforas, formando una única secuencia. De modo que la agencia de viajes hace un uso muy particular de las reofertas.

Para que esta estrategia resulte efectiva, el locutor recurre a las tres estrategias más utilizadas en las ofertas de viajes: la oferta futura, la conminación a la oferta y la oferta explícita. Y las combina dando lugar a tres subestrategias que denominamos subestrategia A, subestrategia B y subestrategia C.

La subestrategia A combina la conminación a la oferta y la oferta futura en la secuencia del acto de habla oferta y supone la subestrategia más numerosa de las reofertas $(57.5 \%)$.

(15) Partez à la découverte du mythe de la Cité Interdite à Beijing et des trésors archéologiques de Xian, jusqu'à la trépidante Shanghai. Ce voyage vous mènera aux riches vestiges de la Chine impériale et millénaire !

(16) La "Perle du Sud" vous charmera par ses palmeraies, ses souks, sa place Jemaâ El Fna et son artisanat. Venez découvrir l'art de vivre marocain!

En (15), la secuencia de oferta se inicia con un enunciado que conmina fuertemente al interlocutor para poder convencerlo (Mansor y Ridruejo, 2014), para después aportar más detalles sobre lo que el viaje supondrá. En (16), la estrategia se produce a la inversa: la agencia comienza la secuencia de oferta mostrándole lo que el viaje le reserva, para exhortarle a continuación a aceptar.

En la subestrategia B, las ofertas están compuestas por dos estrategias: la oferta explícita y la oferta futura. En otras palabras, incorporan enunciados que contienen los verbos performativos proposer, offrir y inviter y enunciados declarativos que expresan la oferta como un acto futuro. Esta subestrategia resulta la segunda más empleada de esta categoría (30.7\%). 
(17) Un must! Une traversée de la CHINE en 15 jours. Nous vous proposons ce circuit très complet de PEKIN à HONG-KONG avec toutes les étapes classiques d'un premier voyage en Chine. En effet, vous trouverez les plus belles étapes de la Chine qui garantissent un vrai dépaysement autour de 7 villes à la beauté attachante. Vous n'avez pas fini d'égrener vos souvenirs au retour!

(18) Habitée par un peuple d'une désarmante gentillesse, la Thaïlande offre au visiteur paysages exotiques, temples scintillants, danses envoûtantes, cités oubliées, minorités ethniques du Triangle d'or, éléphants dans la jungle et plages de sable fin. Ce programme, bien rythmé, vous la fait découvrir dans toute son éclatante diversité et authenticité...

(19) Nous vous invitons à découvrir et déguster le meilleur de la Bourgogne, ses vins, sa culture et son patrimoine. Vous séjournerez pendant 2 nuits à la résidence les Allées du Green, qui bénéficie à la fois du calme de la campagne, au milieu d'un parc verdoyant proche des vignobles de renom (tels que Pommard, Meursault, Nuits St Georges, Gevrey-Chambertin), à seulement $3 \mathrm{~km}$ de Beaune.

En estos ejemplos, nuevamente se ofrece en primer lugar el viaje y el destino vacacional y, después, se hace hincapié en lo que dicho destino puede ofrecer.

Finalmente, la subestrategia $\mathrm{C}$, la menos numerosa de la estrategia de las reofertas (11.8\%), se vale también de dos estrategias: la conminación a la oferta y la oferta explícita. La secuencia de oferta contiene pues enunciados imperativos que le dan inicio seguidos por enunciados que contienen sobre todo los verbos performativos offrir (20) y proposer (21).

(20) Partez au volant de votre $4 \mathrm{X} 4$ de location, en liberté et selon votre rythme. Cet autotour vous offre la sécurité des réservations à chaque étape mais la souplesse d'un voyage en individuel. Au rendez-vous des paysages époustouflants et des sites culturels intéressants ainsi qu'un accueil chaleureux et de superbes plages de sable blanc.

(21) Découvrez l'âme profonde de la Normandie et faites connaissance avec l'Orne. Campagne généreuse, bocage reposant, royaume du cheval l'Orne propose aux amoureux de la campagne mille et un trésors...

Los ejemplos (20) y (21) expresan la intención del anunciante de propiciar un acercamiento con el público y de beneficiarle con un viaje que puede interesarle.

Gracias a esta estrategia, hemos podido confirmar que en francés se insiste en la oferta inicial realizando una reoferta. Destacamos asimismo la cohesión entre la oferta inicial y la reoferta en las tres subestrategias, formando así una única secuencia de oferta. Esto demuestra el interés del anunciante por redactar un mensaje que, además de cohesivo, resulte claro, conciso, efectivo y coherente. La distancia espacio-temporal que existe entre locutor e interlocutor obliga al locutor a redactar mensajes publicitarios que cumplan con esas características para no dar lugar a malas interpretaciones o a ambigüedad. 


\subsection{La oferta explícita}

Tras estas estrategias principales, situamos en nuestro estudio las ofertas explícitas: un procedimiento que incluye enunciados cuyo verbo principal es performativo. A pesar de que resultan menos numerosas que las precedentes (10.7\% de las ofertas de nuestro corpus), muestran, como asume Pohle (2009), que las ofertas pueden emplear otras acciones similares al verbo offrir (22) (49.2\% de las ofertas explícitas), como son en nuestro caso la proposición (23), de uso mayoritario en las ofertas explícitas (37.3\%), o la invitación (24), de uso menor $(13.6 \%)$.

(22) Ce club Lookéa vous offre des vacances de rêve dans un cadre paradisiaque : sable blanc, eaux turquoises, jardin tropical, confort, décoration raffinée et hospitalité thaillandaise...

(23) Au bord du lac Christus, l'hôtel Calicéo propose une pause zen permettant de décompresser, le temps d'un court séjour.

(24) Nous vous invitons à l'émerveillement au cœur de l'un des plus beaux sites d'Europe classé au Patrimoine de l'Unesco, le Mont-St-Michel.

Aunque el uso que se hace de la proposición resulta novedoso: en las ofertas de viajes, adopta la misma fuerza ilocutiva de la oferta, es decir, el acto lo realiza el locutor para el provecho del interlocutor. Esta novedad en el uso del verbo proposer concuerda con la visión que aportan las estrategias mayoritarias sobre el acto de habla oferta de nuestro corpus de ofertas pertenecientes a la publicidad digital de viajes.

\subsection{La presentación de la oferta}

Las ofertas de nuestro corpus también están confeccionadas por enunciados declarativos que contienen presentativos como voici y voilà. En la teoría no están descritos estos enunciados como estrategia para la consecución del acto de ofrecer y, por lo tanto, no constan como ofertas. No obstante, sostenemos que pueden funcionar como ofertas por el contexto comunicativo en el que se producen.

(25) Entre désert et océan Indien, voici le sultanat d'Oman, trésor préservé de la péninsule arabique. À vous de le découvrir!

(26) Profonds canyons, routes bordées de vignobles, villes côtières animées et chutes d'eaux impressionnantes, voilà quelques-unes des surprises qui vous attendent en Afrique du Sud.

En nuestras ofertas, a tenor de estos ejemplos, voici y voilà sirven para realizar el acto de ofrecer ya que pueden atraer la atención del cliente a la oferta y proponerle el viaje. Constituyen otra novedad que nuestro trabajo confiere al estudio del acto de habla oferta. 


\subsection{La oferta posibilitadora}

La oferta posibilitadora, a su vez, es una estrategia compuesta por enunciados declarativos que contienen el verbo modal pouvoir.

(27) Un circuit complet 14 jours/12 nuits, à la découverte du grand ouest américain et où vous pourrez vous promener sur le Colorado ou tenter votre chance au casino. À vous l'ouest des USA et ses paysages mythiques.

(28) Dans ce club rénové entouré d'un parc paysager et situé à seulement $800 \mathrm{~m}$ de la plage, vous pourrez choisir entre une escapade dans la région et farniente au bord de la piscine.

Como vemos en (27) y (28), estas ofertas no instigan al otro a tomar una decisión respecto a ellas, sino más bien muestran como una posibilidad lo que las ofertas procuran al cliente. En un principio, observamos que puede parecerse a la estrategia denominada por Barron (2005) State Permission o a la que Pohle (2009) llama Possibility Statement. La estructura de ambas es muy similar a la de la oferta posibilitadora: State Permission toma la estructura You can VP (29), y, Possibility Statement, la estructura NP can/could VA (30).

(29) Eng. bev.: You can come in any way...

(30) $<$ Ir4B $>$ : You can deal with me, you know?

El ejemplo (29) se produce en una situación en la que el locutor ofrece algo de beber y el interlocutor lo declina y (30), en el contexto de las negociaciones empresariales. A pesar de las similitudes, lo cierto es que no concuerdan con nuestra estrategia. En (29) y (30), la acción que beneficia al interlocutor se puede realizar de manera inminente, mientras que, en la oferta posibilitadora, las actividades descritas en las ofertas de viajes solo se pueden llevar a cabo en un futuro próximo. Este rasgo de la oferta posibilitadora representa una importante novedad de nuestro corpus de ofertas.

\subsection{El anuncio de la oferta}

Y, por último, analizamos la estrategia que denominamos el anuncio de la oferta. Esta recurre a enunciados interrogativos para anunciar al lector la oferta que se va a realizar de forma inminente y captar su atención, apelando a la nostalgia (31), a la disposición (32), al deseo (33) o al sueño (34) del lector.

(31) Nostalgiques d'une période dorée où Istanbul était l'étape mythique d'un voyage de rêve? Partez à la découverte de cette ville aux mille et une facettes...

(32) Vous avez un week-end prolongé? N'hésitez plus et partez à la découverte de la plus belle ville d'Afrique du Sud. 
(33) Envie d'authenticité entre nature et découvertes? Profitez de notre séjour exclusif aux mille couleurs, aux portes de la Camargue; un site unique entre sable et mer, pour admirer toute une faune et une flore protégée: flamants roses, chevaux sauvages, étangs scintillants...

(34) Vous rêvez de l'Est canadien? Voici un séjour dense d'une semaine pour une première expérience réussie à prix serré. Des spectaculaires chutes du Niagara aux baleines de Tadoussac, en passant par Toronto, Québec et Montréal, la plus grande ville francophone après Paris, on ne se lasse pas de ce premier et intense regard sur le Canada.

Si bien estos enunciados interrogativos podrían parecerse a las preofertas por su función de anunciar y prevenir el acto (Tsui, 1994; Kerbrat-Orecchioni, 2000), lo cierto es que no lo mitigan (Ruiz de Zarobe, 2012). Dicho de otro modo, en las ofertas del (31) al (34), el locutor no prepara al interlocutor para prevenirle de un eventual acto verbal: al contrario, dispone de estas secuencias para potenciarle el eventual deseo de lo ofrecido. La secuencia posterior a los enunciados interrogativos se realiza mayoritariamente a través de enunciados imperativos ((31), (32), (33)) (42.1\%) y, en menor número, de otros enunciados como, por ejemplo, aquellos realizados con el presentativo voici (34) (5.3\%). El objetivo de estos últimos es instigar al posible cliente para que realice el viaje o acercarle el viaje. En enunciados imperativos en general incluso se le exhorta explícitamente a aceptar.

Para completar este análisis, estudiaremos a continuación otros rasgos fundamentales de estas estrategias, ligados al acto de habla, que también nos permiten definir la oferta.

\section{Rasgos fundamentales de las estrategias lingüísticas utilizadas en el acto de habla director}

El análisis que hemos realizado en el apartado anterior nos da algunas de las claves necesarias para estudiar otros rasgos importantes de las estrategias lingüísticas utilizadas en nuestras ofertas relativos al acto de habla como son las condiciones de felicidad, la orientación, la realización directa e indirecta y la naturaleza intrínseca del acto de habla oferta (Pohle 2009).

Para analizar las condiciones de felicidad, hemos tenido en cuenta los estudios de autores que definen dichas condiciones para el acto de ofrecer. Roulet (1977) propone seis condiciones para que la oferta se lleve a cabo de manera eficaz que se refieren a la capacidad del locutor, a su disposición, a lo que sabe hacer, al deseo del interlocutor de que el locutor realice la acción, al consentimiento del interlocutor para que el locutor realice la acción y a la utilidad de la acción para el interlocutor. Barron (2003) y Pohle (2009), por su parte, enumeran aquellas condiciones que se utilizan en la oferta. Barron (2003) afirma que en el acto de ofrecer se cumplen las condiciones de contenido proposicional, las condiciones preparatorias, las condiciones de sinceridad y las condiciones esenciales. Pohle (2009) sostiene que en las ofertas empleadas en las negociaciones empresariales se 
cumplen las condiciones de contenido proposicional, las condiciones de sinceridad y las condiciones esenciales.

De las condiciones de felicidad definidas por estos estudios, se cumplen explícitamente en algunas estrategias de nuestro corpus las condiciones de contenido proposicional, las condiciones de sinceridad y las condiciones preparatorias.

En el caso concreto de las primeras, como hemos advertido en el análisis de la oferta futura y la oferta posibilitadora, estas presentan dos nuevas condiciones que definen el acto de ofrecer como:

- Una acción futura del interlocutor

- Una posibilidad para el interlocutor

Además, con respecto a las condiciones de sinceridad, las estrategias o bien muestran explícitamente el compromiso de la agencia con el acto para demostrar que su deseo de que el interlocutor acepte la oferta es sincero, o bien llevan a cabo una segunda oferta, tras la oferta inicial; esto es, en este segundo supuesto el acto de ofrecer no resulta sincero hasta que el locutor no realiza una reoferta (Searle, 1969), como sucede en la estrategia de las reofertas.

Y las condiciones preparatorias del acto también ofrecen algunas novedades porque son específicas de nuestras ofertas de viajes: además del deseo, en el anuncio de la oferta aparecen otras condiciones preparatorias nuevas del acto como el sueño, la disposición o la nostalgia. Se añade una condición inédita en la que lo ofertado está a disposición del interlocutor, condición que se emplea en la oferta posibilitadora.

Las nuevas condiciones de contenido proposicional que incorporan algunas estrategias vienen a confirmar las orientaciones que toman las ofertas: la mayoría de las ofertas de nuestro corpus, exactamente el $44.9 \%$, están orientadas al interlocutor. El locutor entiende que, para alcanzar sus objetivos comerciales, debe centrar todos sus esfuerzos en convencer al interlocutor o a los interlocutores.

(35) L'hôtel St Barth Isle de France est situé au bord d'une des plus belles plages de Saint-Barthélemy, la plage des Flamands, à seulement 5 minutes en voiture de l'aéroport. Les chambres sont disséminées entre plage, colline et jardin tropical. Ici, vous avez rendez-vous avec le luxe au naturel.

(36) La région est propice à la littérature et c'est au Musée de la Comtesse de Ségur à Aube que vous débuterez votre escapade normande. Au détour des routes tranquilles du Perche, faites une halte à Mortagne-au-Perche, cité de caractère et ville de naissance du philosophe Alain où un musée lui est dédié.

Se decanta asimismo por orientar la oferta al locutor y al interlocutor (24.7\%).

(37) Ce circuit hors normes vous offre le lac Titicaca et autres merveilles de la Bolivie sur un plateau andin ! 
(38) Le soleil est bien là, c'est le moment de vous échapper sur l'une des plus belles plages de la Costa Brava à Gérone. L'hôtel Alga $3 *$ vous invite pour un court séjour ensoleillé à petit prix.

Al incluir explícitamente a ambos, comprendemos el papel que toma cada uno de ellos en la oferta: el locutor es el agente de la acción, y el interlocutor, el receptor del viaje. En el caso concreto del locutor, la agencia de viajes se acoge a dos recursos para favorecer las ofertas publicitarias de viajes de Internet y conseguir que el otro empatice con ellas. Por un lado, posiciona al viaje (37) o al alojamiento (38) como sujeto, es decir, recurre a un agente con el rasgo [-humano] (Hurtado González, 2014) o lo que Benveniste (1966) conoce como el desplazamiento del "yo" a la "no persona". Así, da mayor relevancia a la oferta. Y, por otro, en los ejemplos (39) y (40), utiliza el deíctico nous para solidarizarse con el lector y crear un terreno común.

(39) Nous vous proposons un nouveau séjour dans un hôtel-club convivial avec de nombreux équipements pour satisfaire parents et enfants: baignade, repos, animation, activités sportives, bien-être. C'est aussi le point de départ idéal pour découvrir les plus beaux sites de la Crète.

(40) Cet hôtel de ville nous invite au calme et à la détente au cœur de son jardin luxuriant. Et si l'on souhaite mettre de l'action dans notre séjour, on file sur le front de mer où se succèdent boutiques et restaurants.

El desplazamiento de sujetos o el recurso a un agente con el rasgo [-humano] como sujeto se produce igualmente en las ofertas dirigidas únicamente al locutor (4.9\%). En (41), es el país el que realiza el acto y, en (42), es el alojamiento el que lo lleva a cabo.

(41) L'Afrique du Sud, ce "Monde en un seul Pays" offre une grande diversité : la magnifique ville de Cape-Town, le cap de Bonne Espérance, les animaux du Parc Kruger, les canyons du Drakensberg, les villages de la Ruée vers l'Or et les jardins de Pretoria sont autant de visites variées, au fil d'un voyage inoubliable...

(42) Un grand classique de la destination, une ambiance "club des Caraïbes" pour petits et grands. Fidèle à sa philosophie, la chaîne italo-dominicaine Viva Resorts propose un programme d'activités varié, atout majeur de ce complexe qui en fait l'endroit idéal pour passer des vacances animées.

Viendo que estas tres orientaciones resultan potenciales estrategias que benefician a las ofertas, el turoperador también las combina (24.4\%) en un nuevo intento por hacer que este tipo de publicidad de viajes sea lo más atractiva posible.

(43) Un splendide itinéraire qui vous mènera tout autour du Puget Sound, cette immense baie entre Seattle et Victoria. Vous aimerez l'ambiance "cool" de Seattle, le site admirable de Vancouver, le charme de Victoria et les paysages de bout du monde de la péninsule olympique. Sans compter un détour sur la côte Pacifique. Attention : croisements d'ours et des baleines garantis. 
(44) Et si vous partiez en direction du Piémont, magnifique région italienne célèbre pour ses lacs et sa gastronomie? L'hôtel Sirio vous propose une offre qui enchantera aussi bien vos yeux que vos papilles. Séjournez 3 jours et 2 nuits dans un établissement situé en bord de lac, et savourez 1 délicieux dîner pour 2 dans le restaurant de l'hôtel référencé au Guide Michelin.

En (43), la agencia combina un enunciado en el que es el propio viaje el que realiza la oferta (un splendide itinéraire) al lector (vous) con un enunciado orientado únicamente al interlocutor. En (44), la secuencia de oferta está formada por tres enunciados: el primer enunciado está orientado al interlocutor (vous), el segundo al alojamiento (l'hôtel Sirio) y al interlocutor (vous) y el último está orientado nuevamente al interlocutor mediante verbos en imperativo (séjournez, savourez).

En cuanto al tipo de realizaciones, existe gran igualdad en el uso de formulaciones directas (38.7\%) (la conminación a la oferta, la oferta explícita y la subestrategia C de las reofertas), e indirectas (37.5\%) (la oferta futura, la presentación de la oferta y la oferta posibilitadora), además de estrategias que combinan ambas formulaciones (23.8\%) (el anuncio de la oferta y las subestrategias A y B de las reofertas).

A nuestro parecer, los factores no lingüísticos que rodean al acto de habla permiten al locutor mostrar abiertamente o no sus deseos o intenciones. Esto se debe a que las formulaciones directas no se interpretan como imposiciones de dichos deseos o intenciones, sino que sirven para aproximarse al interlocutor. En nuestro corpus, el acto de ofrecer se formula pues directa e indirectamente, en contra de los estudios que asumen que la oferta emplea esencialmente realizaciones indirectas (Roulet, 1977; Conein, 1985, 1986; Ruiz de Zarobe, 2000; Chodorowska-Pilch, 2002, 2003). La realización directa de las ofertas se convierte así en otra de las innovaciones a destacar en nuestro corpus.

Por último, en lo relativo a la naturaleza intrínseca del acto, en nuestra investigación comprobamos que la oferta futura y la oferta posibilitadora potencian el carácter comisivo (32\%); el anuncio de la oferta y la conminación a la oferta, el carácter directivo (28.7\%); la oferta explícita resulta comisivo-directiva (10.7\%); la presentación de la oferta no resalta el carácter comisivo ni directivo (5.5\%), y las reofertas resaltan la naturaleza de las estrategias que incorporan (23.1\%). La naturaleza comisiva y directiva de nuestras ofertas resulta una novedad porque en estudios como el de López Montero (2016), en el que se analizan los actos de habla empleados en la publicidad de la prensa escrita, se utilizan mayoritariamente actos directivos.

En este punto, recalcamos que el carácter directivo no se corresponde con el de los actos directivos prototípicos porque en nuestras ofertas de viajes se exhorta al cliente a aceptar, no se le obliga (Mansor y Ridruejo, 2014). En cuanto al carácter comisivo, el rasgo novedoso de nuestras ofertas es que dicha comisividad está más cercana a la de la promesa que a la de la oferta: las acciones que oferta la agencia únicamente se producen si el interlocutor acepta el viaje. 
Este análisis que acabamos de hacer sobre estos otros rasgos importantes de las estrategias lingüísticas presentes en el acto de habla director nos muestra las novedades que aporta nuestra investigación al estudio del acto de habla oferta y lo actualiza.

\section{Análisis de la modificación externa}

Tras las estrategias del acto de habla director y sus rasgos más importantes, nuestro análisis también se ocupa del estudio de aquellos elementos que acompañan al acto de habla director y completan la secuencia de oferta: la modificación externa.

Entre los modificadores que determina Pohle (2009) para las ofertas en el contexto de las negociaciones empresariales, en nuestras ofertas de viajes están presentes los Expanders ${ }^{2}$ (52.4\%), los Grounders $^{3}$ (5.6\%) y las condiciones explícitas (1.3\%). En este punto, debemos aclarar que, en realidad, un $55.8 \%$ de las ofertas de nuestro corpus incorporan dicha modificación frente al $44.2 \%$ que no la contienen. Además, las ofertas que emplean modificación pueden incluir uno o varios modificadores.

De acuerdo con la definición ofrecida por Pohle (2009) sobre estos modificadores, los Expanders son aquellos que completan la información del acto de habla director; los Grounders, aquellos que justifican su consecución o explican su finalidad, y las condiciones explícitas, aquellas que apelan al gusto del interlocutor para facilitar la consecución del acto verbal. Sin embargo, lo cierto es que toda esta modificación no se limita a esas funciones. Los Expanders, los Grounders y las condiciones explícitas resultan potenciales estrategias de cortesía positiva: favorecen la oferta, le facilitan a la agencia de viajes su labor y consiguen una mejor predisposición del interlocutor hacia lo ofertado.

Los Expanders, sea en la posición que sea (inicial, media, final o inicial y final de la secuencia de oferta), acompañan al acto de habla director para ofrecer toda la información que el locutor entiende que el destinatario requiere para conocer la oferta, cumpliendo con la Máxima de Cantidad de Grice (1979). Esta máxima establece que los hablantes deben ofrecer la información necesaria en los intercambios verbales, pero nunca más de lo necesario.

(45) Remontez le temps au fil du Nil et entrez dans la magie de l'Égypte éternelle. Cette magnifique croisière sur le Nil vous emportera dans le monde mythique des pharaons. C'est une croisière généreuse qui s'offre à vous avec ses visites nombreuses, ses plaisirs à bord de l'un de nos bateaux sélectionnés.

(46) Budapest ville au cœur de l'Europe. Venez découvrir sa culture et séjourner dans l'hôtel Soho au design contemporain, situé à Pest dans l'ancien quartier juif. Il offre une décoration basée sur le métissage savant de styles.

2 Los Expanders son modificadores que aportan información adicional (Pohle, 2009).

3 Los Grounders pueden definirse como Justificadores en español (Real Academia Española, 2019). 
En (45) y (46), la información facilitada por los Expanders contribuye a perfilar la oferta: los lugares que van a poder visitar, los detalles del alojamiento o del tipo de viaje y el programa de actividades. Asumimos que el locutor utiliza los Expanders como procedimiento para hacer que la oferta cautive al interlocutor y la contrate, y así cumplir con sus objetivos comerciales. El uso de este modificador resulta pues muy novedoso.

Los Grounders, como interpretan Brown y Levinson (1987), Barron (2005) y Pohle (2009), ofrecen razones para realizar la oferta en interés del interlocutor.

(47) Le trekking en autonomie est l'une des meilleures façons de découvrir la Mongolie, de rencontrer les nomades, de partager leurs us et coutumes, de s'émerveiller devant les paysages caractéristiques du pays... C'est pourquoi nous vous proposons ce circuit de 13 jours, qui vous donnera un aperçu de ce pays unique.

(48) Situé à l'entrée du complexe Golden 5 city, cet hôtel vous offrira une expérience unique au cœur de beaux jardins grâce à ses services de qualité et ses nombreuses activités.

En estas ofertas, además de justificar su realización mediante causales como c'est pourquoi (47) o grâce à (48), lo que se consigue es que las ofertas resulten muy atractivas a ojos del interlocutor.

Por último, las condiciones explícitas se utilizan sobre todo para mostrar al lector que lo que se está ofreciendo se ajusta a sus preferencias y puede beneficiarle, y para que la oferta no se entienda como una orden o una imposición.

(49) On vivra au rythme oriental dans cet hôtel surplombant l'océan. Et si l'on veut épicer son séjour, on se baladera jusqu'au centre d'Agadir, très proche et très animé. On profite également de son centre de Spa oriental.

(50) Si vous n'avez pas beaucoup de temps, si vous cherchez un dépaysement total, Miami et la route des Keys sur la mer vous comblera.

En (49) y (50), las condiciones explícitas apelan a los gustos del interlocutor o lo que este busca, preparándolo para el momento del acto de ofrecer y los actos de habla directores se muestran como una posible solución a dichos deseos del interlocutor. Entendemos que la condición explícita y el acto de habla director forman una única estrategia para la consecución de la oferta. Además, dichas condiciones están formuladas de forma que el interlocutor difícilmente decline la oferta. El locutor considera todo aquello que pueda interesar al otro para que las ofertas resulten muy atractivas y no se produzcan rechazos.

Es comprensible que la mitad de ofertas de nuestro corpus empleen alguno de estos modificadores para que la oferta se lleve a cabo satisfactoriamente, aunque tienen mayor representatividad los Expanders que los Grounders y las condiciones explícitas. El locutor, a causa del espacio limitado de las páginas web, se decanta más por incluir información sobre el viaje que haga la oferta más completa y ensalce el viaje, que por justificarla o facilitar su consecución. 


\section{Conclusiones}

En este estudio, hemos tomado como referencia trabajos enmarcados en la teoría de los actos de habla, así como trabajos específicos de la oferta para analizar el acto de ofrecer en un corpus de 550 ofertas de viajes en francés tomadas de las páginas web de 43 agencias de viajes. Extraemos de este análisis importantes novedades respecto a la naturaleza del acto y a las estrategias lingüísticas mediante las que se lleva a cabo.

Descubrimos que la oferta publicitaria de viajes de Internet contiene algunas similitudes, pero sobre todo diferencias con las ofertas que se producen en interacciones verbales cara a cara. La oferta perteneciente a la publicidad de viajes de Internet se realiza mediante estrategias que inciden en la voluntad del cliente, insisten en lo ofertado, llevan a cabo el acto de manera más explícita, o presentan, anuncian o muestran la oferta como una posibilidad, estrategias todas ellas propias de este tipo de publicidad (el de viajes) y que están sujetas a ciertas condiciones para que el anunciante pueda cumplir con sus objetivos comerciales. Posee tres orientaciones diferentes, pero con procedimientos particulares. Se realiza directa e indirectamente, pero abundando en la realización directa como novedad. Favorece la naturaleza comisiva y directiva, pero se trata de una comisividad y una directividad diferentes a las descritas por los trabajos enmarcados en la teoría de los actos de habla y los trabajos específicos sobre la oferta. Y se modifica externamente, pero principalmente con un modificador (los Expanders) de empleo novedoso.

En definitiva, esta investigación sobre el acto de habla oferta en la publicidad digital de viajes en francés ofrece una nueva perspectiva al estudio de este acto verbal, al incluir un contexto y un medio revolucionarios y al realizar algunas consideraciones que no se tenían de él hasta el momento. A tenor de lo analizado, consideramos, además, que puede inspirar nuevos estudios sobre actos de habla enmarcados en el contexto de Internet, imprescindible ya en la comunicación diaria entre hablantes, para renovar esta línea de investigación pragmática.

\section{Referencias bibliográficas}

Aijmer, Karin. 1996. Conversational routines in English: Convention and Creativity. Londres, Longman.

Austin, John Langshaw. 1975. How to do things with words. Cambridge, Massachusetts, Harvard University Press.

BACH, Kent \& Robert HaRnish. 1979. Linguistic Communication and speech acts. Massachusetts, The MIT Press.

Barron, Anne. 2003. Acquisition in Interlanguage Pragmatics: Learning How to Do Things with Words in a Study Abroad Context. Amsterdam/Philadelphia, Benjamins. 
Anales de Filología Francesa, n. ${ }^{\circ}$ 28, 2020

El ACTO DE HABLA OFERTA EN LA PUBLICIDAD DIGITAL DE VIAJES EN FRANCÉS

BARron, Anne. 2005. "Offering in Ireland and England" in BARRON, Anne \& Klaus Peter SCHNEIDER (eds. e introd.). The Pragmatics of Irish English. Berlín, Mouton de Gruyter, 141-176.

Benveniste, Émile. 1966. Problèmes de linguistique générale. Paris, Gallimard.

Bhatia, Vijay Kumar. 1993. Analysing Genre: Language use in Professional Settings. Londres, Longman.

Bolaños, Alicia, María Jesús Rodríguez, Lydia Bolaños \& Luis Losada. 2004. Análisis del discurso electrónico: convenciones genéricas, lingüísticas y funcionales. Las Palmas de Gran Canaria, Universidad de las Palmas de Gran Canaria.

Blum-Kulka, Shoshana, Julianne House \& Gabriele Kasper. 1989. Cross-Cultural Pragmatics: Requests and apologies. Norwood, NJ, Ablex Publishing.

Brown, Penelope \& Stephen Levinson. 1987. Politeness. Some universals in language usage. Cambridge, Cambridge University Press.

Chodorowska-Pilch, Mariana. 2002. "Las ofertas y la cortesía en el español peninsular" in Placencia, María Elena \& Diana BRAVO (eds.). Actos de habla y cortesía en español. Munich, Lincom Europa, 21-36.

Chodorowska-Pilch, Mariana. 2003. "Las ofertas corteses en el español peninsular" in Bravo, Diana (ed.). Actas del $1^{\text {er }}$ Coloquio del Programa Édice. Estocolmo, Stockholm University, 306-314.

Conein, Bernard. 1985. "Actes de parole et organisation des relations sociales: comment fait-on des offres au téléphone ?" in Réseaux, vol. 3, n 13, 111-126.

Conern, Bernard. 1986. "Conversation et interaction sociale: analyse de séquences d'offre et d'invitation" in Langages, $\mathrm{n}^{\circ}$ 81, 111-120.

Grice, Herbert Paul. 1979. "Logique et conversation" in Communications, no 39, 57-72.

HANCHER, Michael. 1979. "The classification of cooperative illocutionary acts" in Language in Society, vol. 8, $\mathrm{n}^{\circ}$ 1, 1-14.

Hurtado González, Silvia. 2014. "Titulares de actos de habla en la prensa hispana con el orden declaración-declarante" in ELUA, $\mathrm{n}^{\circ} 28,251-266$.

Kerbrat-Orecchioni, Catherine. 2000. "Énonciation et argumentation dans les annonces publicitaires" in ADAM Jean-Michel \& Marc BONHOMME (eds.). Analyses du discours publicitaire. Toulouse, Éditions Universitaires du Sud, 113-142.

Kerbrat-Orecchioni, Catherine. 2004. "Il fait vraiment chaud aujourd'hui ! Vous voulez boire quelque chose ? Le 'travail des faces' dans l'échange initié par une offre" in AucHLIN, Antoine, Marcel Burger, Laurent Filliettaz, Anne Grobet, Jacques Moeschler, Laurent PERRIN, Corinne ROSSARI \& Louis de SAUSSURE (eds.). Structures et discours. Mélanges offerts à Eddy Roulet. Québec, Nota bene, 417-432.

López de Lizaga, José Luis. 2015. "Pedir, exigir, ordenar, coaccionar. Searle y Habermas sobre la fuerza ilocucionaria de los actos de habla" in Estudios de lingüística del español, $\mathrm{n}^{\circ}$ $36,411-430$. 
Mansor, Nor Shahila \& Emilio Ridruejo Alonso. 2014. “Análisis pragmático de los actos de habla directivos en la publicidad malaya" in Lenguas Modernas, $\mathrm{n}^{\circ}$ 44, 67-79.

López Montero, Rosberly. 2016. "Actos de habla en el lenguaje publicitario escrito del periódico rural La Voz del Pacífico" in RECIAL, Áreas Letras, vol.7, n 10, 1-17.

PÉrez Hernández, Lorena. 2001a. “The act of offering” in PÉrez Hernandez, Lorena. Illocution and cognition: a constructional approach. Logroño, Universidad de La Rioja, $307-$ 320.

PÉrez Hernández, Lorena. 2001b. "The directive-commissive continuum" in Miscelánea: a journal of English and American studies, $\mathrm{n}^{\circ} 23,77-98$.

PoHLe, Stephanie (2009). I tell you what we could do, we could say, cut it to a hundred and ninety-five, and offer you a significant discount on breakfast- Expressing Commitment in Business Discourse: An Empirical Analysis of Offers in Irish English Negotiations. Tesis doctoral, Universidad de Bonn.

Roulet, Eddy. 1977. "Étude des réalisations directes et indirectes de l'acte d'offre en français parlé" in Studi italiani di linguistica teorica ed applicata, $\mathrm{n}^{\circ}$ 6, 525-539.

Ruiz De Zarobe, Leire. 2000. “Ofrecimiento y cortesía en francés” in E.L.U.A., no 14, 185197.

Ruiz De Zarobe, Leire. 2000-2001. "Estrategias de invitación en español e imagen social de los hablantes: Un estudio empírico" in Pragmalingüística, vol. 8-9, 261-278.

Ruiz De Zarobe, Leire. 2012. "Learning-offers on the Internet in Spanish and French" in Ruiz De Zarobe, Leire \& Yolanda Ruiz De Zarobe (eds.). Speech Acts and Politeness across Languages and Cultures. Berna, Peter Lang, 173-195.

SchneIder, Klaus Peter. 2003. Diminutives in English. Tübingen, Niemeyer.

SEARLE, John. 1969. Speech acts: an essay in the philosophy of language. Cambridge, Cambridge University Press.

SEARLE, John. 1979. Expression and meaning: studies in the theory of speech acts. Cambridge, Cambridge University Press.

Tsui, Amy. 1994. English conversation. Oxford, Oxford University Press.

Real Academia Española. 2019. "Diccionario de la lengua española". España, Real Academia Española <https://www.rae.es/> [29/04/2020].

\section{Anexo: enlaces a las páginas web de las agencias de viajes}

www.planetanoo.com

www.kuoni.fr

www.partirenvoyages.com

www.directours.com 
Www.promovacances.com

www.nouvelles-frontieres.fr

www.arvel-voyages.com

www.philibertvoyages.fr

www.marmara.com

www.atypik-travel.com

www.fr.lastminute.com

www.opodo.fr

www.sensationsdumonde.com

www.agence-de-voyages.com

www.selectour.com

www.bourse-des-voyages.com

www.promosejours.com

www.afatvoyages.fr

www.ailleurs.com

www.belambra.fr

www.stivoyages.fr

www.voyages.carrefour.fr

www.travelzoo.com/fr

www.tangka.com

www.vestiges-tours-online.com/fr/

www.escaporne.fr

www.le-voyage-autrement.com

www.tropicalement-votre.com

www.aventuria.com

www.tematis.com

www.akaoka.com

www.route-voyages.com

www.mon-tour-motos.com

www.lafrancaisedescircuits.com

www.heliades.fr

www.voyamar-vacances.com

www.unmondeadeux.com

www.croisitour.com

www.voyage-moto.com

www.corse-a-moto.com

www.terreentiere.com

www.vacancestransat.fr

www.makila.fr 